\section{Conceitos de acesso à saúde}

\author{
Raquel Maia Sanchez ${ }^{1}$ \\ e Rozana Mesquita Ciconelli ${ }^{1}$
}

Como citar: Sanchez RM, Ciconelli RM. Conceitos de acesso à saúde. Rev Panam Salud Publica. 2012;31(3):260-8.

\section{SINOPSE}

Esse artigo descreve quatro dimensões de acesso à saúde disponibilidade, aceitabilidade, capacidade de pagamento $e$ informação, correlacionado-as aos seus indicadores e tecendo considerações sobre a complexidade do conceito de acesso. Para a revisão desses conceitos foram pesquisadas as bases de dados PubMed/MEDLINE, LILACS, SciELO e World Health Organization Library \& Information Networks for Knowledge (WHOLIS). Veículos de grande circulação, como a revista The Economist, o jornal The Washington Post $e$ os arquivos da rede $B B C$ também foram pesquisados. $O$ conceito de acesso à saúde modificou-se ao longo do tempo, tomando uma forma mais complexa. As primeiras análises, datadas da década de 1970, sugeriam uma forte relação do acesso com o aspecto geográfico (disponibilidade) e financeiro (capacidade de pagamento). A literatura mais recente procura abordar aspectos menos tangíveis, como os aspectos cultural, educacional e socioeconômico, incorporando o elemento aceitabilidade nas análises. A literatura mostra ainda que ter informação está na base do acesso à saúde, estando essa noção associada aos conceitos de empoderamento e de letramento para a tomada de decisões de saúde. Concluiu-se que a melhoria do acesso à saúde $e$ a garantia de uma maior equidade não serão obtidas com ações cujo foco se limite aos sistemas de saúde. Em vez disso, dependem de ações inter-setoriais e politicas sociais e econômicas que permitam dissipar diferenças de renda $e$ educação.

Palavras-chave: acesso aos serviços de saúde; equidade em saúde; políticas públicas de saúde.

Universidade Federal de São Paulo, Escola Paulista de Medicina, São Paulo (SP), Brasil. Correspondência: Raquel Maia Sanchez, raquel.msanchez@gmail.com
As desigualdades em saúde são um tema central das discussões que envolvem políticas públicas em diversas nações, no mundo todo. As desigualdades no acesso à saúde estão profundamente ligadas aos cenários políticos nacionais e internacionais, às políticas sociais e econômicas e a fenômenos como globalização e crescimento econômico (1).

A definição do que seja o acesso à saúde e a criação de um modelo que possa mensurar esse acesso têm sido objeto de muito interesse, já que esses aspectos são fundamentais para o desenvolvimento de planos e metas sustentáveis no setor saúde. Entretanto, ao longo do tempo, o conceito de acesso à saúde tornou-se mais complexo, com a incorporação de aspectos de mais difícil mensuração.

Em 1974, Aday e Andersen (2) conceituaram o acesso aos serviços de saúde tomando por base as características da população e a disponibilidade organizacional e geográfica do sistema de saúde; por outro lado, já definiam o acesso como uma ideia mais política do que operacional. Ronald e Newman (3), em 1973, avaliaram os determinantes individuais que interferem no acesso à saúde, como, por exemplo, características da população (renda, cobertura do seguro saúde, atitudes frente ao cuidado com a saúde, estrutura social) ou das instituições de saúde; Penchansky e Thomas (4) argumentaram que o acesso poderia ser avaliado através de indicadores de resultado da passagem do indivíduo pelo sistema de saúde (por exemplo, a satisfação do paciente) e definiram o acesso como o grau de interação entre os clientes e o sistema de saúde. Donabedian (5), que utiliza o termo acessibilidade em vez de acesso, descreveu dois aspectos: sócio-organizacional e geográfico. Para esse autor, os atributos sócio-organizacionais incluem a condição social, cultural, educacional ou econômica do indivíduo, que termina por influenciar significativamente o acesso à saúde. A acessibilidade geográfica, por outro lado, refere-se a uma função do tempo e do espaço, como a distância física entre o usuário e os serviços. Donabedian argumentou que o termo "acessibilidade" abrange uma gama maior de significados, pois está mais relacionado à oferta de serviços de um modo que responda às necessidades da população do que à simples disponibilidade dos recursos em um determinado tempo e espaço.

Mais tarde, McIntyre e Mooney incorporaram à noção de acesso à saúde a liberdade para o uso dos serviços: os atributos que definem o indivíduo, como seu nível educacional, sua bagagem cultural e crenças, sua condição social e econômica, entre muitos outros atributos, e a relação do indivíduo com o sistema de saúde farão com que esse indivíduo seja mais ou menos "livre" para utilizar o sistema, o que 
se traduzirá em melhores ou piores condições de acesso à saúde. Além disso, para McIntyre e Mooney, a informação que o profissional de saúde e o paciente possuem é um importante determinante da qualidade da interação entre o sistema de saúde e o indivíduo (1).

Segundo a ótica da Organização Mundial da Saúde (OMS) (6), a promoção de saúde é definida como o processo pelo qual as pessoas são habilitadas a melhorar a sua saúde e aumentar o controle sobre ela. Essa perspectiva deriva da premissa de que o indivíduo é capaz, por um lado, de compreender suas aspirações e satisfazer suas necessidades, e, por outro lado, de modificar e cooperar com o ambiente. A saúde é, dessa forma, vista como um recurso para a vida cotidiana. É um conceito positivo, que enfatiza os recursos sociais e pessoais. O conceito de promoção de saúde, nesse caso, extrapola o acesso físico ao sistema de saúde e engloba outros aspectos, como o conhecimento do indivíduo sobre suas próprias necessidades e aspirações.

Assim, ao longo do tempo, o conceito de acesso à saúde passou a incorporar dimensões que refletem aspectos menos tangíveis do sistema e da população que o utiliza. Atualmente, as principais características do acesso à saúde são resumidas em quatro dimensões: disponibilidade, aceitabilidade, capacidade de pagamento e informação (1). Essas dimensões podem ser avaliadas por indicadores de processos e resultados, que auxiliam na determinação da existência de equidade ou desigualdade no acesso à saúde, conforme descrito por Aday e Andersen (2).

Nesse contexto, o objetivo deste artigo foi descrever as dimensões de acesso à saúde, correlacionado-as aos seus indicadores e tecendo considerações sobre a complexidade do conceito de acesso.

\section{MÉTODOS}

Foram revisadas as seguintes bases de dados: PubMed/MEDLINE, LILACS e SciELO, sem determinação de um período definido. Para a busca no PubMed, os operadores booleanos e os descritores utilizados foram: "social justice" AND "health services accessibility" AND "healthcare disparities", "costbenefit analysis" AND "social justice" AND "health services accessibility" e "quality of health care" AND "social justice" AND "health services accessibility".

Na busca realizada na base de dados LILACS, utilizou-se o descritor "acesso aos serviços de saúde". $\mathrm{Na}$ base de dados SciELO, os seguintes descritores foram utilizados: "access equity" OR "access to health care" OR "access to health information" OR "access to healthcare" OR "access to healthcare services" OR "access to medical services", utilizando-se o índice títulos de artigos como filtro para a busca.

Além da busca nas bases de dados, realizou-se uma busca manual nas listas de referências dos artigos selecionados. Foram incluídos artigos de revisão e artigos que buscavam definir ou contextualizar o conceito de acesso e equidade em saúde. Aqueles artigos que não atenderam a esses critérios de inclusão foram ex- cluídos do estudo. Foram pesquisados também capítulos de livros relacionados ao tema de acesso à saúde.

A base de dados oficial da OMS, World Health Organization Library $\mathcal{E}$ Information Networks for Knowledge (WHOLIS), disponível em www.who.org, também foi utilizada. Veículos de grande circulação como a revista The Economist (www.economist.com), o jornal The Washington Post (www.washingtonpost.com) e os arquivos da rede BBC também foram pesquisados utilizando-se a expressão health care reform (reforma da saúde).

Os veículos de comunicação brasileiros também foram explorados através da ferramenta de busca Google News (disponível em http://news.google. com); algumas notícias consideradas relevantes foram selecionadas e incluídas na análise.

\section{RESULTADOS}

De 152 artigos e demais documentos selecionados, 34 foram citados na presente análise.

A figura 1 representa as dimensões de acesso à saúde, cujas características são descritas a seguir, e exemplifica os indicadores que podem ser utilizados para avaliar essas dimensões.

\section{Disponibilidade}

A dimensão do acesso definida como disponibilidade constitui-se na representação da existência ou não do serviço de saúde no local apropriado e no momento em que é necessário. Essa dimensão engloba, de forma ampla, a relação geográfica entre as instituições físicas de saúde e o indivíduo que delas necessita, como distância e opções de transporte. Os recursos de transporte dos profissionais de saúde, bem como sua disposição para mobilizar-se até o indivíduo que necessita do serviço de saúde, também podem ser considerados nesta análise. O nível pelo qual os serviços de saúde se ajustam às necessidades dos indivíduos, como, por exemplo, o horário de funcionamento (especialmente para adultos que trabalham) ou o momento em que os serviços são necessários (emergência), também faz parte do elemento disponibilidade (1).

A disponibilidade compreende ainda a relação entre tipo, abrangência, qualidade e quantidade dos serviços de saúde prestados. Essa interação é influenciada pelas políticas adotadas pelos serviços de saúde, pelo número, competência e experiência dos profissionais de saúde contratados em uma determinada instituição de saúde, pelas regulações que regem as interações de saúde e pela existência de equipamentos e insumos médicos, entre outros (1).

A disponibilidade é importante pois representa um conceito físico e geográfico: reflete a entrada no sistema de saúde (5) e possibilita a mensuração de vários indicadores de processos e de resultados (2). Os indicadores de processo refletem as características do sistema de distribuição e da população em risco que afetam a efetivação da entrada no sistema e o grau de satisfação dos consumidores com o sistema. As 
FIGURA 1. Dimensões de acesso à saúde e indicadores

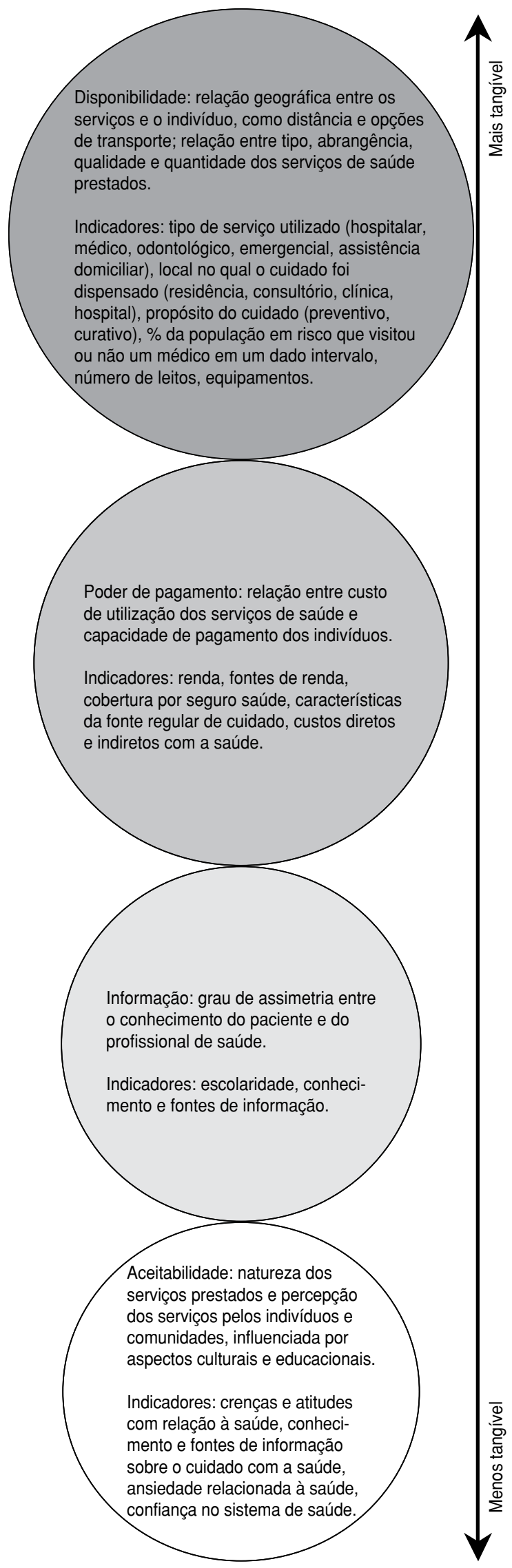

medidas de distribuição e volume podem derivar da análise de dados como número de médicos, de leitos hospitalares e de ambulâncias por unidade populacional ou área geográfica. No que tange à organização, variáveis mensuráveis que afetam a entrada no sistema de saúde podem incluir tempo de viagem, tempo de espera médio para o agendamento de consultas, tempo de espera médio para o atendimento, bem como tempo médio para a chegada da ambulância. Os indicadores de resultado, ou seja, utilização e satisfação, refletem os produtos finais das políticas de saúde em relação ao acesso. Medidas de utilização incluem: as especificações e o tipo de serviço utilizado (hospitalar, médico, odontológico, emergencial, assistência domiciliar); o local no qual o cuidado foi dispensado (residência, consultório, clínica, hospital); o propósito do cuidado dispensado (preventivo, curativo); o intervalo de tempo envolvido (porcentagem da população em risco que visitou e não visitou um médico em um dado intervalo de tempo, número médio de visitas a um médico em um dado intervalo de tempo); e a continuidade, mensurada através do número de diferentes profissionais de saúde contatados em um dado episódio de doença (2).

Travassos e Martins (7) apontaram para a tendência no deslocamento do conceito de acesso do seu eixo de entrada nos serviços para os resultados dos cuidados de saúde. A alta hospitalar após condições hospitalares evitáveis compõe uma medida válida do acesso ao cuidado primário efetivo e pontual, segundo Gusmano et al. (8). O cuidado primário reduz a probabilidade de hospitalização por enfermidades tratadas efetivamente fora do ambiente hospitalar. Tais condições incluem pneumonia, insuficiência cardíaca congestiva, diabetes e asma. $\mathrm{O}$ acesso ao cuidado especializado, por outro lado, pode ser medido por meio das taxas de altas hospitalares após procedimentos que requerem um especialista e que são indicados para condições que podem não representar ameaça imediata à vida, como, por exemplo, cirurgias de reconstrução da mama após o tratamento do câncer, revascularização coronária, entre outros. Travassos e Martins (7) citaram ainda a abordagem da OMS, que sugeriu um novo indicador de avaliação de desempenho dos sistemas de saúde, chamado "cobertura efetiva", definida como a proporção da população que necessita de um determinado procedimento de saúde e que recebeu de forma efetiva esse procedimento.

Apesar do grau de detalhamento, a dimensão da disponibilidade não é suficiente para garantir o acesso, nem para avaliá-lo.

\section{Capacidade de pagamento (affordability)}

Essa dimensão do acesso compreende a relação entre o custo de utilização dos serviços de saúde e a capacidade de pagamento dos indivíduos e engloba conceitos relacionados ao financiamento do sistema de saúde. Podem ser consideradas nessa análise: despesas com consultas médicas, medicamentos e testes diagnósticos, entre outros; outras despesas diretas, 
como transporte ou alimentação especial; e despesas indiretas, como perda de renda ou produtividade em consequência do tempo de espera no atendimento, por exemplo (1).

A capacidade de pagamento pode incorporar o subconceito de capacidade financeira (ability-to-pay), que envolve, por exemplo, a elegibilidade dos indivíduos a benefícios de diversos tipos de financiamento de saúde. Também fazem parte dessa definição: a quantidade, temporalidade e frequência do salário de cada indivíduo em um dado domicílio; a redução de gastos e a poupança, que podem gerar capacidade para arcar com pagamentos relacionados à saúde; o número de bens que um indivíduo ou grupo de indivíduos possuem; a extensão e natureza das redes sociais, que podem permitir que os indivíduos se organizem para mobilizar fundos; o acesso a crédito; entre outros (1).

Segundo Aday e Andersen (2), são indicadores do poder de pagamento a renda e as fontes de renda, a cobertura por seguro saúde (tipo de pagador, extensão da cobertura, forma de pagamento) e características da fonte regular de cuidado (tempo de viagem médio, tempo de espera para atendimento).

McIntyre e Mooney (1) sugeriram alguns elementos que poderiam ser utilizados como indicadores de capacidade de pagamento, como:

- custos diretos com saúde, como, por exemplo, preços de consultas, custos de testes diagnósticos, custos de medicamentos;

- custos indiretos, como custos com transporte e alimentação especial;

- custos indiretos, como perda de produtividade em decorrência do tratamento;

- elegibilidade dos indivíduos para a cobertura de planos de saúde ou o sistema público de saúde;

- renda, poupança, elegibilidade a crédito.

\section{Informação}

A informação é essencial para a compreensão de cada uma das dimensões do acesso. Estar bem informado é resultado do processo de comunicação entre o sistema de saúde e o indivíduo. Permeando a discussão, é importante ressaltar a noção do empoderamento (empowerment) para tomar decisões bem informadas sobre o uso dos serviços de saúde (1).

A informação imperfeita e as incertezas são atributos importantes da economia da saúde, conforme descrito por Arrow (9). A assimetria de informação desigualdade na distribuição de informação entre os atores do sistema de saúde - é uma das formas pelas quais os sistemas de saúde podem ser analisados e melhorados, segundo Thiede e McIntyre (10). Quando há assimetria de informação, uma das partes do sistema de saúde detém informações significativas, enquanto que a outra parte não possui informações suficientes para fazer uso do sistema de forma adequada ou suficiente para atender às suas reais necessidades.

A percepção individual de saúde e doença, a subjetividade que permeia os critérios de escolha no contexto da saúde e as preferências individuais são determinantes da escolha realizada pelo indivíduo em relação à sua saúde. A subjetividade ora mencionada pode ser traduzida como o número de escolhas possíveis percebido pelo indivíduo dada a informação possuída por ele. O número de escolhas possíveis percebido pelo indivíduo é frequentemente menor do que o número real de opções disponíveis, devido à ausência de conhecimento das opções por esse indivíduo (10). O conhecimento precário ou ausente impede o indivíduo de realizar as escolhas mais adequadas para a sua necessidade, efeito descrito por Thiede e McIntyre como falta de empoderamento.

A informação pode determinar a lacuna entre a oportunidade de utilização e a real utilização dos serviços de saúde. Alguns autores acreditam que a informação é o cerne do debate da equidade, considerando que o processo de empoderamento das pessoas para que estas façam escolhas em relação ao cuidado com a sua saúde seja um objetivo fundamental das políticas de saúde em uma sociedade democrática (10).

Aday e Andersen (2), em sua análise estruturada sobre os indicadores de processo e resultado para mensurar o acesso à saúde, exemplificaram o conhecimento e fontes de informação como indicadores de processo. Segundo Thiede e McIntyre (10), o nível adequado de informação para que as pessoas possam tomar decisões em saúde pode ser atingido através da educação. De acordo com Perlow (11), o letramento em saúde é um componente importante na eliminação de disparidades em saúde.

\section{Aceitabilidade}

A aceitabilidade compreende a natureza dos serviços prestados e o modo como eles são percebidos pelos indivíduos e comunidades. A aceitabilidade é a dimensão menos tangível do acesso e, portanto, a mais difícil de ser quantificada e detectada. A relação entre as atitudes dos profissionais de saúde e dos indivíduos, influenciadas por idade, sexo, etnia, idioma, crenças culturais, condição socioeconômica, entre outros, define a aceitabilidade. A interação entre as expectativas dos profissionais de saúde e as expectativas dos pacientes também influencia a aceitabilidade dos serviços de saúde: expectativas dos profissionais de que os pacientes respeitarão sua condição e aderirão ao tratamento prescrito; expectativas dos pacientes de que serão tratados com respeito pelos profissionais de saúde, de que os profissionais ouvirão atentamente a descrição dos sintomas, conduzirão um exame minucioso, explicarão as doenças e discutirão alternativas de tratamento; e as expectativas dos pacientes em relação à organização do sistema de saúde. Um ponto chave no conceito de aceitabilidade é o respeito mútuo entre profissionais de saúde e usuários dos serviços (1).

Dentre os indicadores de processo sugeridos por Aday e Andersen (2), alguns podem ser classificados como indicadores de aceitabilidade, como as variáveis que influenciam as mudanças nas decisões relativas às 
políticas de saúde, ou fatores de predisposição, quais sejam: crenças e atitudes com relação à saúde, conhecimento e fontes de informação sobre o cuidado com a saúde, ansiedade com relação à saúde. As variáveis imutáveis que guardam relação com a aceitabilidade incluem idade, sexo, estado civil, comportamento de saúde anterior, educação, etnia, tamanho e composição da família, religião e mobilidade residencial.

Travassos (12) indicou a confiança no sistema de saúde como um elemento essencial para a equidade no acesso. Por outro lado, a falta de confiança no sistema de saúde pode gerar uma barreira ao acesso. A confiança, bem como o respeito mútuo, também foram destacados por McIntyre e Mooney (1) quando da abordagem do elemento aceitabilidade.

\section{DISCUSSÃO}

Embora ainda exista considerável debate sobre o conceito de acesso à saúde, a maior parte da literatura concorda que acesso não equivale à simples utilização do serviço de saúde. $\mathrm{O}$ acesso tem sido descrito como a oportunidade de utilização dos serviços em circunstâncias que permitam o uso apropriado dos mesmos. Padrões de utilização devem ser analisados com cautela, pois não constituem um elemento indicador do acesso per se, embora possam auxiliar na avaliação da equidade em saúde (1).

Mooney (13) conceituou o acesso à saúde de acordo com a natureza e a extensão das barreiras ao cuidado conforme percebidas por diferentes grupos. $\mathrm{O}$ autor propõe uma revisão do acesso com base em um contexto de justiça social. O conceito de justiça social foi pontuado por Swartz (14) como uma distribuição igualitária de recursos sociais, como nutrição, habitação, saúde e educação. Conforme a revisão realizada por Mooney, a distribuição de renda também é considerada por muitos como um determinante da desigualdade em saúde.

Mechanic (15) afirmou que as características de recursos e serviços não são suficientes para determinar a entrada ou não entrada no sistema de saúde. Deve-se levar em conta também a potencial determinação (willingness) do paciente de buscar o cuidado, assim como a aceitabilidade (1), que, conforme definido anteriormente, envolve o respeito mútuo e a proximidade entre as crenças do profissional de saúde e dos leigos. Outro elemento da aceitabilidade é a natureza do engajamento paciente-profissional de saúde e do diálogo que se estabelece nessa interação, a qual sofre influência da habilidade do paciente e de sua disposição para se engajar em um diálogo com os profissionais de saúde. Grupos pertencentes a classes sociais mais altas podem ter maior habilidade para se engajar em um diálogo com o profissional de saúde, por exemplo. O elemento final da aceitabilidade é relacionado aos arranjos organizacionais do sistema de saúde e ao modo como eles moldam a resposta do paciente ao cuidado. Esses arranjos e respostas podem, inicialmente, permitir ou evitar o acesso do paciente à completa gama de serviços necessários à saúde. Um fator organizacional fundamental é a confiança do paciente no profissional de saúde (1).

A aceitabilidade e os problemas de confiança influenciam a decisão do paciente e a experiência adquirida e são mais propensos a afetar de forma desvantajosa os grupos socialmente marginalizados. Em particular, os problemas de aceitabilidade ligados à confiança estão relacionados a (1):

- indisposição do paciente em relatar seu histórico médico, dificultando o diagnóstico e o tratamento;

- taxas menores de utilização dos serviços secundários e terciários e menores taxas de intervenção em relação à necessidade;

- adesão limitada ao tratamento ou não atendimento ao seguimento do tratamento, particularmente no cuidado crônico;

- menor número de relatos de saúde espontâneos.

Sorkin et al. (16) afirmam que as minorias raciais e étnicas nos Estados Unidos são desproporcionalmente afetadas pela baixa qualidade nos cuidados com a saúde. Estudos mostram que diferenças raciais e étnicas no acesso à saúde e no recebimento de cuidado médico e de tecnologias que salvam vidas podem ser resultado tanto de fatores relacionados ao sistema como também do comportamento individual do médico. Por exemplo, a raça/etnia pode influenciar a interpretação dos relatos do paciente pelo médico e, finalmente, influenciar a decisão clínica.

O gênero também exerce influência importante sobre o acesso aos cuidados com a saúde. Em algumas sociedades, como o Paquistão, as mulheres não podem tomar decisões por si próprias, nem mesmo procurar pelo cuidado com a saúde sem a permissão e o auxílio do chefe da família; essas mulheres receberão potencialmente menos cuidados da família durante o tratamento do que os homens (17). Por outro lado, em sociedades ocidentais, pode ocorrer o inverso: as mulheres tendem a mostrar melhor predisposição para o uso dos serviços de saúde do que os homens (18).

McIntyre e Mooney (1) mencionam que equidade e acesso são, em parte, culturalmente determinados, e debatem ainda o conceito da arrogância ocidental, assim como o fato de a linguagem e a cultura das discussões internacionais sobre saúde e equidade serem dominadas pelo pensamento ocidental. A justiça social pode ser interpretada diferentemente por pessoas diferentes em cenários diferentes; portanto, uma definição é necessária para que possa ser operacionalizada com base em critérios mensuráveis. Em uma sociedade multicultural, os construtos de saúde, acesso e equidade também são variáveis culturais. Os autores acreditam ainda que não podemos pressupor que todas as barreiras ao acesso serão percebidas igualmente por todos, mesmo que sejam objetivamente as mesmas. Em resumo, pode-se avaliar a aceitabilidade como um aspecto cultural, educacional e comportamental que influencia o acesso ao cuidado com a saúde. É bem menos tangível que a disponibilidade, porém não menos importante. 
A discussão de desigualdades em saúde traz à tona, necessariamente, o conceito de equidade no cuidado com a saúde. Entretanto, a equidade pode ser representada de diversas formas. A equidade horizontal é definida como tratamento igual para indivíduos iguais. Por outro lado, a equidade vertical se define como tratamento desigual, porém equilibrado, de indivíduos desiguais (19). O estabelecimento de conceitos diferentes para a mesma condição na equidade vertical deve-se ao entendimento, por diversos autores, de que indivíduos diferentes possuem, via de regra, necessidades diferentes. Portanto, a equidade vertical consegue, na maior parte das vezes, representar melhor o conceito de justiça social do que a equidade horizontal.

Woodward e Kawachi (20) afirmam que a equidade pode ser traduzida como a eliminação de desvantagens atribuíveis a fatores que vão além do controle do indivíduo. Por exemplo, na Nova Zelândia, a saúde precária da população indígena Maori está ligada à história da colonização nos anos de 1800 e 1900. As causas dos problemas de saúde incluem a alienação da terra e a supressão da cultura e língua Maori. Pode-se argumentar que a desigualdade atual entre a população Maori e não Maori é um problema de equidade, em decorrência desse poderoso e histórico fator de predisposição, o qual se encontra fora do controle das pessoas que experienciam um excesso de doença.

Diferenças atribuíveis a escolhas individuais, como, por exemplo, escolhas relacionadas a crenças pessoais e aspectos culturais, não seriam, essencialmente, iniquidades em saúde. Historicamente, a preocupação com a equidade em saúde vem aumentando e propiciando avanços no campo das políticas sociais. No Brasil, a criação do Sistema Único de Saúde (SUS), em 1988, propôs universalidade, integralidade e equidade no acesso à saúde como direito de todo cidadão brasileiro (21).

Ao longo dos anos, após a Segunda Guerra Mundial, muitas nações ocidentais realizaram as reformas necessárias para implementar um sistema de saúde universal. Com a chegada de novas tecnologias médicas efetivas, parte dos países vislumbrou um sistema de financiamento da saúde que garantisse o acesso igualitário, independentemente da capacidade de pagamento do usuário. Entretanto, o acesso universal não se transformou em equidade em saúde. Mais recentemente, tem-se observado que alguns determinantes de saúde repousam fora do escopo e da responsabilidade direta do sistema de saúde. Distribuição desigual de determinantes de saúde, como moradia, condições de trabalho, ambiente seguro, alimentação saudável, acesso ao esporte e estilos de vida, requer políticas inter-setoriais e preventivas (22).

Os custos com a saúde e a existência de despesas de saúde que precisam ser assumidas pelos próprios indivíduos criam barreiras financeiras que impedem as pessoas de procurar e receber cuidados com a saúde. A OMS (23) encoraja os países a desenvolverem sistemas de financiamento de saúde com cobertura universal. O maior desafio para alcançar uma cobertura universal é encontrar maneiras de substituir o atual sistema de dispêndio individual por algum sistema de pré-pagamento.

Gusmano et al. (24) compararam o sistema de saúde da Inglaterra (cobertura universal, pagador único) com o francês (sistema multipagador com cobertura universal) e o norte-americano (sistema multipagador, caracterizado por amplas diferenças na cobertura de saúde). O resultado colocou em dúvida a noção de que o número de pagadores influencia a capacidade do sistema de saúde de fornecer acesso igualitário aos seus residentes. $\mathrm{O}$ artigo mostrou que o número de pagadores é menos importante do que a capacidade do sistema de assegurar o acesso a serviços primários e especializados. Os achados de Gusmano et al. (24) indicam que a cobertura universal isoladamente não é suficiente para eliminar as iniquidades geográficas do acesso ao cuidado. Entretanto, também identificou-se que a cobertura universal pode reduzir disparidades marcantes no acesso ao cuidado primário, por reduzir as barreiras financeiras ao cuidado com a saúde.

Em 1999, Gunning-Schepers e Stronks (22) previram que os países do ocidente enfrentariam um rápido envelhecimento de suas populações, com aumento simultâneo das necessidades e custos do sistema de saúde. A viabilidade financeira do acesso universal seria ameaçada, e seria necessário o estabelecimento de prioridades.

Gunning-Schepers e Stronks (22) sugerem um modelo para a definição de prioridades com base no fato de que os recursos escassos não suportariam uma cobertura universal por tempo indeterminado. A premissa mais importante é a opção da sociedade por uma delimitação clara da responsabilidade coletiva, determinada principalmente pelas consequências de delegar a responsabilidade de certos serviços ao indivíduo; desta forma, a sociedade aceitaria que o acesso ao cuidado fosse negado àqueles com inabilidade de pagar pelo serviço. $\mathrm{O}$ acesso universal seria mantido para serviços considerados como sendo de responsabilidade coletiva, para o qual um sistema de fundos seria implementado (sistema de seguro social compulsório ou sistema baseado em impostos).

Áreas como a prevenção podem requerer uma ação coletiva, como, por exemplo, o controle do ar, considerando que o indivíduo não pode fazê-lo. A prevenção de doenças infecciosas também deve ser classificada como uma responsabilidade coletiva, que necessita do estabelecimento de programas de vacinação. De maneira similar, o atendimento emergencial e intervenções que salvam a vida, os quais são realizados independentemente da capacidade de pagar, são responsabilidades coletivas.

Williams (25) afirma que a acessibilidade é mais custosa em algumas áreas do que em outras. Uma vertente proposta pelo autor afirma que a redução das iniquidades deveria levar em conta a experiência de saúde ao longo da vida dos indivíduos. O autor propõe um ponto de partida para nortear as discussões 
sobre a redução das iniquidades, procurando entender quão grande é o sacrifício que a população geral estaria disposta a fazer para tornar a distribuição de saúde menos desigual.

Woodward e Kawachi (20), por sua vez, acreditam que é possível reduzir as desigualdades em saúde sem reduzir a saúde da população em geral. Por exemplo, intervenções estruturais e ambientais podem potencialmente afetar a população de forma mais ampla, como ocorre com a fluoretação da água, que traz benefícios e reduz as desigualdades em termos de saúde bucal. Os autores descrevem quatro argumentos favoráveis à redução das iniquidades em saúde, quais sejam: iniquidades são injustas, iniquidades afetam a todos, iniquidades são evitáveis e intervenções para a redução das iniquidades em saúde são custo-efetivas. Ações de redução das desigualdades em saúde geralmente produzem externalidades positivas (efeito spillover) e, via de regra, são custo-efetivas - embora, como apontam os próprios autores, nem sempre os efeitos das externalidades positivas sejam tão claros, como no caso das doenças coronarianas. Entretanto, há uma crescente gama de estudos que sugerem que ações de redução das desigualdades sociais e econômicas que repousam sobre a distribuição desigual das doenças trará benefícios.

O nível de informação em geral, o qual está relacionado com o nível educacional dos indivíduos, determina, de forma significativa, o acesso individual aos recursos sociais e, portanto, aos cuidados com a saúde. Em uma situação ideal, a desigualdade de informações entre médicos e pacientes não deveria ser um problema: o paciente espera que o médico utilize o seu conhecimento da melhor forma possível, buscando o resultado mais favorável. Por outro lado, o desequilíbrio entre o conhecimento detido pelo médico e o paciente resulta na impossibilidade de o paciente exigir determinado padrão no cuidado com a sua saúde (9).

Perlow (11) descreve a acessibilidade como "capacidade para obter". Esse autor discute o letramento em saúde, definindo-o como o nível no qual os indivíduos têm a capacidade de obter, processar e entender informações básicas de saúde e serviços necessários para tomar decisões apropriadas em saúde. Em outras palavras, o nível educacional do indivíduo, que se traduzirá em letramento em saúde, define a sua capacidade de entender as suas próprias necessidades em saúde e, por consequência, procurar pelo cuidado, assim como tomar decisões bem informadas com relação à sua saúde e bem-estar. Baixas taxas de letramento têm sido relacionadas a resultados adversos na saúde, incluindo hospitalizações, algumas doenças crônicas e aumento dos custos com saúde.

A teoria da comunicação distingue diferentes níveis de efetividade de informação (26). Segundo Thiede e McIntyre (10), o nível no qual a informação à saúde se torna efetiva e empodera as pessoas para suas escolhas com relação às oportunidades para utilizar serviços de saúde depende de uma ampla gama de determinantes. A efetividade da informação pode ser influenciada por diversas características no lado do receptor, como sua condição socioeconômica, mas também depende da relação sociocultural entre os atores que estão trocando a informação.

Cabe lembrar a influência da mídia na educação em saúde. Campanhas de comunicação em massa têm sido utilizadas para modificar o comportamento em saúde das populações, utilizando-se a mídia como veículo de comunicação. Tais campanhas têm focalizado temas como tabagismo, prevenção de doenças do coração, uso de álcool e drogas ilícitas, diagnóstico e prevenção do câncer, doenças sexualmente transmissíveis e outros. Muitas campanhas objetivam afetar indivíduos por meio do aspecto cognitivo e de respostas emocionais e podem afetar o processo de tomada de decisão do indivíduo. A probabilidade de sucesso das campanhas de comunicação em massa aumenta quando o comportamento alvo é um episódio isolado - por exemplo, diagnóstico ou vacinação - em vez de um hábito, como escolhas alimentares, exposição ao sol ou atividade física (27).

\section{CONSIDERAÇÕES FINAIS}

O conceito de acesso pode variar ao longo do tempo, à medida que as sociedades evoluem e novas necessidades surgem. Atualmente, o acesso à saúde é cada vez mais discutido em termos de justiça social e de equidade. $\mathrm{O}$ aumento inexorável dos custos com a saúde em virtude da incorporação de novas tecnologias tem contribuído para acirrar as discussões. Muitos pesquisadores concordam, no entanto, que o acesso à saúde é um conceito de múltiplas dimensões, composto por fatores financeiros e não financeiros.

A discussão acerca do impacto das desigualdades sobre o acesso aos diferentes níveis de atenção também merece consideração. A questão do acesso à informação tem sido debatida por alguns autores. Observou-se que o baixo nível de educação em saúde é determinante na redução do acesso à prevenção em saúde (28). No campo da atenção primária, Sambala et al. (29) sugeriram que o foco na atenção primária é uma abordagem importante para a melhoria dos sistemas de saúde, visando a assegurar o acesso adequado aos serviços e aos medicamentos. Com relação à atenção especializada, a incorporação de novas tecnologias nos sistemas de saúde de países com cobertura universal tem chamado a atenção dos tomadores de decisão em saúde. Ferraz sugere que o processo de avaliação de novas tecnologias em saúde deve ser iniciado pela definição clara das prioridades do sistema de saúde (30).

Um dos panos de fundo da campanha presidencial de Barack Obama nos Estados Unidos foi justamente a reforma da saúde. Os Estados Unidos não possuem um sistema de saúde com cobertura universal, como ocorre em outros países desenvolvidos. Os indivíduos são responsáveis por adquirir seu próprio seguro saúde. As exceções são os programas Medicare e Medicaid. Medicare é um programa de seguro saúde 
para pessoas com 65 anos de idade ou mais, pessoas com alguma deficiência e menos de 64 anos e pessoas de qualquer idade com doença renal em estágio terminal (31). O Medicaid é um programa de seguro saúde para pessoas de baixa renda e alguns grupos com necessidades especiais. Entretanto, de acordo com o censo norte-americano, estima-se que 46,3 milhões de pessoas nos Estados Unidos, de uma população de 300 milhões de habitantes, não possuíam um plano de saúde em 2008 (32). O debate em torno da questão do acesso se intensificou à medida que os custos em saúde para os indivíduos foram aumentando dramaticamente e culminou com a reforma do sistema de saúde em março de 2010.

No âmbito da América Latina, as mudanças que se produziram nos sistemas de saúde podem ser atribuídas ao processo de globalização como variável determinante. A transição das sociedades latinoamericanas de um modelo de desenvolvimento para outro globalizado envolveu a reestruturação dos processos de produção e políticas de ajuste necessárias às novas condições de competitividade internacional. A partir da década de 1980, as reformas nos sistemas de saúde deveriam ser aceleradas no sentido de expandir a cobertura, visando maior equidade, propiciar viabilidade fiscal e financeira e melhorar a eficiência, a qualidade e a satisfação dos usuários (21).

Quanto ao Brasil, o país se prepara para avançar mais uma posição no ranking das economias, porém ainda enfrenta problemas relacionados ao subdesenvolvimento, como o acesso à saúde e a má distribuição de renda (33).

As Metas de Desenvolvimento do Milênio da Organização das Nações Unidas (ONU) incluem o acesso a medicamentos essenciais nos países em desenvolvimento até 2015. Outros aspectos do acesso à saúde são compreendidos nas Oito Metas de Desenvolvimento do Milênio, como a redução da fome, a redução da mortalidade infantil, a melhoria da saúde materna, o combate a HIV / AIDS, malária, tuberculose e outras doenças, e a redução da proporção de pessoas sem acesso a água potável e saneamento básico. Os relatórios apontam para uma melhoria dos indicadores de saúde para cada um dos objetivos estabelecidos, demonstrando uma tendência de redução das desigualdades em saúde (34).

A extensão das iniquidades em saúde tem sido documentada por diversos pesquisadores. Essas iniquidades resultam de uma estratificação social e de desigualdades políticas que rodeiam o sistema de saúde. A renda, a condição social e cultural, condições de habitação e emprego, bem como outros fatores, como raça e sexo, são importantes. As desigualdades também podem ser fruto da forma como os sistemas de saúde criam barreiras aos usuários, gerando problemas na disponibilidade, qualidade e custo dos ser- viços e até na forma como a prática clínica é exercida. Diferenças na vulnerabilidade e exposição, aliadas à desigualdade no acesso à saúde, retroalimentam a desigualdade social e de saúde continuamente.

A definição de acesso, calcada em quatro elementos principais, denominados disponibilidade, aceitabilidade, capacidade de pagamento e informação, cada vez mais se confunde com o conceito de equidade em saúde. A maior parte das análises e pesquisas convergem para uma reflexão comum: o problema do acesso à saúde e, por consequência, da equidade em saúde, precisa ser confrontado através de ações intersetorias e transversais a todas as áreas de governo, abrangendo políticas sociais e econômicas, permitindo melhor distribuição de renda, fortalecimento da cidadania, melhores condições de educação e habitação, e não somente através de ações circunscritas ao nível de atuação dos sistemas de saúde.

\section{ABSTRACT}

\section{The concepts of health access}

This article describes four dimensions of health access-availability, acceptability, ability to pay and information-correlating these dimensions to indicators and discussing the complexity of the concept of access. For a study of these four dimensions, searches were conducted using the PubMed/MEDLINE, LILACS, SciELO, and World Health Organization Library \& Information Networks for Knowledge (WHOLIS) databases. Large-circulation media vehicles, such as The Economist, The Washington Post, and the BBC network were also searched. The concept of health access has become more complex with time. The first analyses, carried out in the 1970s, suggested a strong emphasis on geographical (availability) and financial (ability to pay) aspects. More recently, the literature has focused on less tangible aspects, such as cultural, educational, and socioeconomic issues, incorporating the element of acceptability into the notion of health access. The literature also shows that information provides the starting point for access to health, in association with health empowerment and literacy for health care decision-making. The study concludes that improvements in access to health and the guarantee of equity will not be achieved by initiatives focusing on health care systems alone, but rather will depend on intersectoral actions and social and economic policies aimed at eliminating income and education differences.

Key words: health services accessibility; equity in health; health public policy. 


\section{REFERÊNCIAS}

1. McIntyre D, Mooney G, eds. The economics of health equity. New York: Cambridge University; 2007.

2. Aday LA, Andersen R. A framework for the study of access to medical care. Health Serv Res. 1974;9(3):208-20.

3. Ronald A, Newman JF. Societal and individual determinants of medical care utilization in the United States. Milbank Mem Fund Q Health Soc. 1973;51(1): 95-124.

4. Penchansky R, Thomas WJ. The concept of access: definition and relationship to consumer satisfaction. Med Care. 1981; 19(2):127-40.

5. Donabedian A. Aspects of medical care administration: specifying requirements for health care. Cambridge: Harvard University; 1973.

6. World Health Organization. A discussion document on the concept and principles of health promotion, Copenhagen, 9-13 July 1984. Disponível em: http:// www.who.int/healthpromotion/mile stones_yellowdocument.pdf Acessado em 15 de fevereiro de 2010.

7. Travassos C, Martins M. Uma revisão sobre os conceitos de acesso e utilização de serviços de saúde. Cad Saude Publica. 2004;20(2):190-8.

8. Gusmano MK, Weisz D, Rodwin VG. Achieving horizontal equity: must we have a single-payer health system? J Health Polit Policy Law. 2009;34(4): 617-33.

9. Arrow K. Uncertainty and the welfare economics of medical care. Am Econ Rev. 1963;53(5):941-73.

10. Thiede $M$, McIntyre D. Information, communication and equitable access to health care: a conceptual note. Cad Saude Publica. 2008;24(5):1168-73.

11. Perlow E. Accessibility: global gateway to health literacy. Health Promot Pract. 2010;11(1):123-31.

12. Travassos C. Fórum: equidade no acesso aos serviços de saúde. Cad Saude Publica. 2008;24(5):1159-61.
13. Mooney G. Is it not time for health economists to rethink equity and access? Health Econ Policy Law. 2009;4 (Pt 2):209-21.

14. Swartz O. On social justice and political struggle. Hum Nat Rev. 2004;4:152-63.

15. Mechanic D. Public expectations and health care: essays on the changing organization of services. New York: WileyInterscience; 1972.

16. Sorkin DH, Ngo-Metzger Q, De Alba I Racial/ethnic discrimination in health care: impact on perceived quality of care. J Gen Intern Med. 2010;25(5):390-6.

17. Shaikh BT, Hatcher J. Health seeking behaviour and health service utilization in Pakistan: challenging the policy makers. J Public Health (Oxf). 2004;27(1):49-54

18. Pinheiro RS, Viacava F, Travassos C, Brito AS. Gênero, morbidade, acesso e utilização dos serviços de saúde no Brasil. Cienc Saude Coletiva. 2002;7(4):687-707.

19. Mooney G, Jan S. Vertical equity: weighting outcomes? or establishing procedures? Health Policy. 1997;39(1):79-87.

20. Woodward A, Kawachi I. Why reduce health inequalities? J Epidemiol Community Health. 2000;54(12):923-9.

21. Cordeiro H. Descentralização, universalidade e eqüidade nas reformas da saúde. Cienc Saude Coletiva. 2001;6(2): 319-28.

22. Gunning-Schepers LJ, Stronks K. Inequalities in health: future threats to equity. Acta Oncol. 1999;38(1):57-61.

23. World Health Organization. Sustainable health financing, universal coverage and social health insurance. Genebra: WHO; 2005

24. Gusmano MK, Weisz D, Rodwin VG. Achieving horizontal equity: must we have a single-payer health system? J Health Polit Policy Law. 2009;34(4): 617-33.

25. Williams A. Thinking about equity in health care. J Nurs Manag. 2005;13(5): 397-402.

26. Dervin B. Libraries reaching out with health information to vulnerable popu- lations: guidance from research on information seeking and use. J Med Libr Assoc. 2005;93(4 Suppl):S74-80.

27. Wakefield MA, Loken B, Hornik RC. Use of mass media campaigns to change health behaviour. Lancet. 2010; 376(9748):1261-71.

28. Sanders LM, Shaw JS, Guez G, Baur C, Rudd R. Health literacy and child health promotion: implications for research, clinical care, and public policy. Pediatrics. 2009;124(Suppl 3):S306-14.

29. Sambala EZ, Sapsed S, Mkandawire ML Role of primary health care in ensuring access to medicines. Croat Med J. 2010; 51(3):181-90.

30. Ferraz MB. Dilemas e escolhas do sistema de saúde. Economia da saúde ou saúde da economia? Rio de Janeiro: Medbook; 2008

31. U.S. Department of Health and $\mathrm{Hu}$ man Services, Centers for Medicare and Medicare Services. Medicare and you 2010. Disponível em: www.medicare. gov Acessado em 12 de maio de 2010.

32. BBC News. Q\&A: US healthcare reform. Disponível em: http://news.bbc co.uk/1/hi/8160058.stm Acessado em 15 de fevereiro de 2010

33. BBC Brasil. Disponível em: http://www. bbc.co.uk/portuguese/noticias/2011/ 12/111229 Acessado em 8 de janeiro de 2012.

34. World Health Organization. Millennium Development Goals: progress towards the health-related Millennium Development Goals. Fact sheet no. 290. Genebra: WHO; 2009. Disponível em: http://www.who.int/mediacentre/ factsheets/fs290/en/index.html Acessado em 15 de fevereiro de 2011.

Manuscrito recebido em 26 de novembro de 2010 Aceito em versão revisada em 14 de junho de 2011. 